\title{
Histological findings in pseudomembranous trigonitis
}

\author{
L. HENRY AND M. FOX
}

From the University Department of Pathology and the Department of Urology, The Royal Hospital, Sheffield

SYNOPSIS Pseudomembranous trigonitis presents in females with the urethral syndrome and a characteristic lesion restricted to the trigone of the bladder. The histology shows 'vaginal metaplasia' of the epithelium together with underlying oedema and vascular dilatation, but no significant inflammation. Thirty-one such cases are included in the study. The possible role of oestrogen stimulation is discussed, together with a suggestion that the trigone has an embryological derivation distinct from the remainder of the bladder, allowing the trigonal epithelium to respond to oestrogenic stimulation in a manner denied to the vesical transitional epithelium.

The 'urethral syndrome' is one of the causes of urgency and frequency of micturition in the female, which may be distressing at times. While this may be due to a number of factors, a certain proportion of these women have a normal urine, which is sterile on culture, and a characteristic lesion of the trigone on cystoscopy. Biopsy reveals a metaplasia of the trigonal epithelium which comes to resemble that of the vagina. This condition was first described by Heymann (1905) as 'trigonal cystitis' and has since passed under a variety of names, including pseudomembranous trigonitis, urethrotrigonitis, granular trigonitis, and vaginal or squamous metaplasia of the trigonal epithelium. In a survey of this condition endoscopic biopsy has been performed in 31 cases and the histological findings are described here. Three male patients are also included, two with a papillary transitional-cell carcinoma of bladder and one on oestrogen therapy for carcinoma of the prostate.

\section{Clinical Findings}

The 31 patients were all women with urgency and frequency of micturition. Their ages ranged from 15 to 73 with a mean of 38 years. Nine patients were postmenopausal. The urine was normal on routine testing and sterile on culture. Clinical examination was negative.

\section{Cystoscopic Findings}

On endoscopy, the changes are confined to the Received for publication 7 January 1971. trigone, the remainder of the bladder being normal, although the lesion may extend round the ureteric orifices or into the upper urethra. There is an abnormal greyish, heaped-up mucosa with a serpigenous margin and a cuff of surrounding hyperaemia. The lesion is raised above the surrounding surface. Bleeding may occur during cystoscopy.

A biopsy was taken in each case through a resectoscope, and divided in two with a sterile scalpel. One portion was cultured, this being negative in each case, and the other was processed for histology.

\section{Histological Findings}

The tissue was fixed in either Bouin's fluid or in $4 \%$ formaldehyde with routine processing to wax. Sections were cut at $5 \mu$ and stained with haematoxylin and eosin, PAS with and without diastase, Best's carmine and Masson's trichrome. Holmes' stain for nerve fibres was used on a few specimens.

All of the biopsies had certain features in common. The epithelium showed a non-keratinizing squamous metaplasia and was thicker than the normal transitional epithelium (Fig. 1). The cells were vacuolated and contained much glycogen, giving an appearance similar to vaginal epithelium. The term 'vaginal metaplasia' was applied to this change. The edge of the lesion showed a sudden transition from the normal to the vaginal type of epithelium (Fig. 2). The lamina propria showed a marked venous dilatation (Fig. 3). There was no thrombosis but four cases showed minimal polymorph margination. The arteries were normal with no fibrinoid change. Considerable oedema was present in this layer 
Fig. 1 The epithelium of the trigone showing squamous metaplasia to a 'vaginal' type of epithelium. Haematoxylin and eosin $\times 125$.

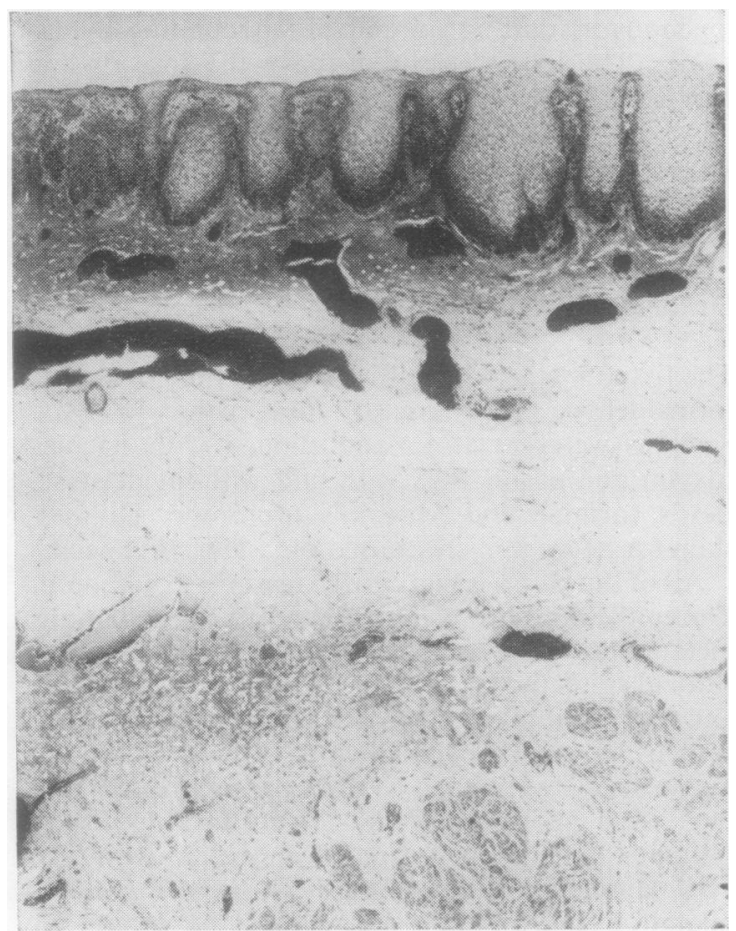

Fig. 2 The edge of a lesion showing the sudden transition to the 'vaginal' type of epithelium. $H \& E . \times$ 100.

Fig. 3 The lamina propria shows oedema and venous dilatation. There is a little fibrosis extending into the underlying muscle. $H \& E . \times 30$.

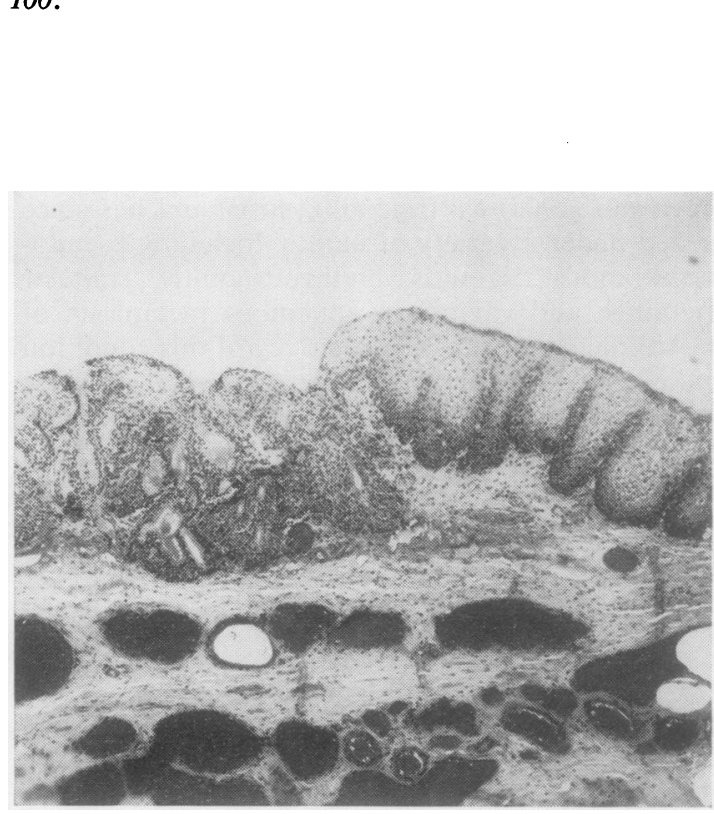

Fig. 4 Pseudomembranous trigonitis in association with cystitis glandularis. The glandular epithelium does not

show the vaginal metaplasia. $H \& E . \times 40$. 


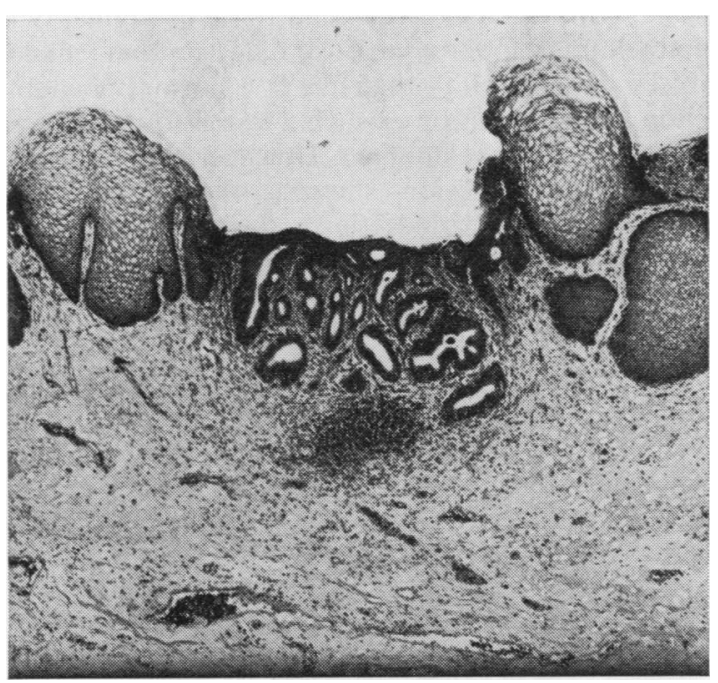

Fig. 5 Vaginal metaplasia and cystitis glandularis in the trigone of a male. There is no underlying oedema or vascular dilatation. $H \& E . \times 30$.

(Fig. 3). Only minimal cellular infiltration was seen and nine cases showed no inflammatory exudate at all. The remainder showed scattered lymphocytes or minor focal collections of these cells but no true lymphoid follicles. Occasional plasma cells were seen in 13 cases, eosinophils in two, and polymorphs in one case. However, in each instance the cellular infiltration was mild and barely significant in contrast to the findings of Corrin, Mayor, and Moore (1963) in cases of bladder-neck obstruction. No abnormality was present in the nerve supply. In four cases the lamina propria showed a significant fibrosis and in each of the six cases where the muscle of the bladder wall was included there was a slight degree of interstitial fibrosis (Fig. 3). In addition to these changes two cases showed cystitis cystica and six cases areas of cystitis glandularis (Fig. 4). The condition, therefore, must be distinguished from the pure forms of cystitis cystica and glandularis, and from non-specific inflammation where there may be an inflammatory pseudomembrane or a true keratinizing squamous metaplasia. No paraurethral ducts were observed such as those described by Moore (1960) as the 'female prostate'. 'Vaginal metaplasia' of the trigonal epithelium has been seen in three male patients, two undergoing a transurethral resection of a papillary transitional-cell carcinoma of bladder and one on oestrogen therapy for carcinoma of prostate. In these the changes were confined to the epithelium and the other components-oedema and vascular dilatation-were lacking (Fig. 5). They could not, therefore, be classified as 'pseudomembranous trigonitis'.

\section{Discussion}

The name 'pseudomembranous trigonitis' is not appropriate in a pathological sense, since there is no significant inflammation present nor any inflammatory pseudomembrane. However, its clinical usage has some justification. The vaginal metaplasia will give rise to a white plaque with the appearance of a membrane. The oedema will produce a raised lesion and the vascular dilatation gives the impression of an inflammatory process. However, other names are equally unsatisfactory. 'Squamous metaplasia' would include keratinizing forms not seen in the present cases while 'vaginal metaplasia' fails to take account of the oedema and vascular elements of the lesion. The clinical name will therefore be retained.

The condition has been described by various authors (Heymann, 1905; Ryall, 1929; Orr, 1933; Fowler, 1936; Cifuentes, 1947; Ney and Ehrlich, 1955; Clarke and Gherardi, 1962). All of these refer to young menstruating women with no cases before the menarche and a decreasing incidence after the menopause. The disease has not been reported in males. 'Vaginal metaplasia' of the trigonal epithelium can be seen in normal females, but the oedema and vascular elements are not present (Streitz, 1963). Tyler (1962) found the change in 12 of 14 premenopausal women and 17 of 34 postmenopausal but none in stillbirths, newborn infants, or children. Similar figures are given by Cifuentes (1947).

This restricted incidence in the absence of any infection may indicate that the condition represents a response of the trigonal epithelium to female sex hormones, the remainder of the bladder epithelium lacking this potentiality. Tyler (1962) found that cyclical changes occur during menstruation in the epithelium of the trigone and urethra, in parallel with the vaginal epithelium. The trigonal epithelium is more atrophic in postmenopausal women. Streitz (1963) found that oestrogens caused thickening and squamous metaplasia of epithelium derived from the urogenital sinus and that oestrogen administration caused the lesion of pseudomembranous trigonitis to increase in size. Experimentally Burns (1941) has shown a similar effect in the young opossum. Oestrogens are known to produce squamous metaplasia in the prostatic ducts when administered for carcinoma of that organ, and the case of Tyler (1962) and that reported here indicate that even in the male there is some capacity of the trigonal epithelium to respond to this hormone.

The restriction of the lesion to the trigone and upper urethra would suggest that the trigone may 
have an embryological derivation differing from the remainder of the bladder. The embryology of the trigone is in some dispute but it is probable that this area is mesodermal in origin, the remainder of the bladder being endodermal. The Wolffian (mesonephric) duct communicates with the urogenital sinus and the ureter arises as a diverticulum from its lower end. Distal to the origin of the ureter, the duct becomes dilated (Brockis, 1952) and is incorporated into the developing bladder. The Wolffian ducts and ureters then open into the bladder separately. Growth occurs at the supero-lateral margins of the ostium of the mesonephric ducts forcing the ureters laterally and forming the trigone between these and the ducts, which now lie together at the base of the bladder. In this theory, therefore, the trigone is Wolffian in origin. However, Burns (1941) and Streitz (1963) have shown that the epithelium of the urogenital sinus itself is sensitive to the action of ocstrogens. Thus the epithelium showing vaginal metaplasia would have migrated into the endodermal bladder from the urogenital sinus unless the trigone itself has a specific derivation from the sinus. Development of the trigone from the Mullerian system would explain the capacity of the epithelium to respond to oestrogens but there is no direct proof of such derivation. During development the Mullerian and Wolffian ducts are opposed, are enclosed in the same connective-tissue sheath (Gruenwald, 1941), and may, rarely, communicate (Tyler, 1962). Thus it is possible for a Mullerian component to contribute to the trigone. Weiss and Dykhuizen (1967) support this view by recording a case of anomalous vaginal insertion into the bladder.

It is certain, however, that the trigone is anatomically distinct from the remainder of the bladder and it may be that its epithelium does have the capacity to respond to female hormones. This may occur either by metaplasia of the transitional epithelium or areas of epithelium may represent embryonic rests pos- sessing this capacity. The occurrence of vaginal type $\frac{\stackrel{D}{*}}{5}$

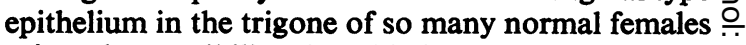
raises the possibility that this is a normal phenomenon and the relation of such a lesion to the clinical $\stackrel{\text { ? }}{+}$ symptoms may be doubted. However, when there is an established lesion showing the oedema and vascular dilatation in addition to the epithelialchanges with a characteristic cystoscopic appearance, it is $\triangle$ probable that the lesion must be included in the in symptom complex in an aetiological capacity.

Mr D. E. Bradey and Mr J. V. Larway gave technical and photographic assistance.

References

Brockis, J. G. (1952). The development of the trigone of the bladder with a report of a case of ectopic ureter. Brit. J. Urol., 24. 192-200.

Burns, R. K.. Jr. (1941). Origin and differentiation of epithelium of $\mathcal{G}$ the urogenital sinus in the opossum. Proc. Soc. exp. Biol. 음 (N.Y.), 47, 106-108.

Cifuentes, L. (1947). Epithelium of vaginal type in the female trigone. J. Urol., 57, 1028-1037.

Clarke, B. G., and Gherardi, G. J. (1962). Urethrotrigonitis or epidermidization of the trigone of the bladder. J. Urol., 87, 545-548. 응

Corrin, B., Mayor, D., and Moore, T. (1963). The pathology of bladder neck obstruction in the female patient. J. Urol., 90, 专 434-439.

Fowler, H. A. (1936). Pseudomembranous trigonitis with observations $\vec{\theta}$ on bladder dysfunction in the female. Urol. cutan. Rev., 40, $\neg$ 156-163.

Gruenwald,P. (1941). Relation of growing Müllerian duct to Wolffian duct and its importance for genesis of malformations. Anat. Rec., 81, 1-19.

Heymann, A. (1905). Die cystitis trigoni der Frau. Zbl. Krankh. Harn.-u. SexOrg., 16, 422-433.

Moore, T. (1960). The female prostate: Bladder neck obstruction in 응 women. Lancet, 1, 1305-1309.

Ney, C. , and Ehrlich, J. C. (1955). Squamous epithelium in the trigone $\overrightarrow{\vec{T}}$ of the human female urinary bladder. J. Urol., 73, 809-819.

Orr, L. (1933). Chronic pseudomembranous trigonitis. Sth. med. J. (Bgham, Ala.), 26, 359-361.

Ryall, E. C. (1929). Pseudo-membranous trigonitis. Brit. J. Urol., 1 , 254-257.

Streitz, J. M. (1963). Squamous epithelium in the female trigone. $J$. Urol., 90, 62-66.

Tyler, D. E. (1962). Stratified squamous epithelium in the vesical trigone and urethra: findings correlated with the menstrual 3 cycle and age. Amer. J. Anat., 111, 319-325.

Weiss, J. M., and Dykhuizen, R. F. (1967). An anomalous vaginal insertion into the bladder. J. Urol., 98, 610-612. 